\title{
THEORY OF STRONGLY CORRELATED SYSTEMS IN THE LIMIT OF LARGE ON-SITE COULOMB REPULSION
}

\author{
Yu.A. Izyumov \\ Institute for Metal Physics, Ural Division of the Russian Academy of Sciences \\ Kovalevskaya 18, 620219 Ekaterinburg, Russia
}

A strongly correlated electronic system is studied within the framework of the $t-J$ model with the help of diagram technique for the Hubbard $X$-operators. The summing of some series of diagrams is suggested, corresponding to the following three types of approximations: the generalized random phase approximation, the mean field approximation, and the low density approximation. In generalized random phase approximation the dynamic magnetic susceptibility of paramagnetic phase is calculated, and boundaries of stability of this phase are found on the plane of Hamiltonian parameters and electron concentration $n$. It is shown that at some critical concentration $n_{c}$ the system undergoes a crossover from the itinerant magnetism $\left(n<n_{\mathrm{c}}\right)$ to the magnetism with localized magnetic moments $\left(n>n_{c}\right)$. Simultaneously, the system transfers from the Fermi-liquid behaviour to the regime of strong electron correlations with nonquasiparticle states. In mean field approximation equations for the order parameters were derived and phase transitions temperatures in a ferromagnetic and antiferromagnetic phases were obtained. In low density approximation a state of the saturated ferromagnetism was investigated and a critical concentration $n_{\mathrm{s}}$ was calculated when the nonsaturated ferromagnetism follows the saturated ferromagnetism.

PACS numbers: $75.10 . \mathrm{Lp}, 75.30 . \mathrm{Cr}$

\section{The $t-J$ model in terms of the Hubbard $X$-operators}

In the theory of strongly correlated electronic systems the $t-J$ model has an important meaning. The model assumes electrons moving over a lattice by hopping from one site to a neighbour site with the matrix element $t$ provided each site should not be occupied by two electrons. Simultaneously electrons on two neighbour sites interact by exchange forces characterized by the antiferromagnetic exchange integral $J$. This model can be considered as a limit case of the Hubbard model [1] with large on-site Coulomb repulsion $U \gg t$. Then by the excluding double occupation of all sites one can come to the strongly correlated electron 
systems with exchange interaction $J=4 t^{2} / U \equiv \kappa t$, where $J \ll t$. The Hamiltonian of the model contains only two parameters $t$ and $J$ and is written as

$$
\mathcal{H}=t \sum_{i j \sigma}\left(1-n_{i,-\sigma}\right) C_{i \sigma}^{+} C_{j \sigma}\left(1-n_{j,-\sigma}\right)+J \sum_{i j}\left(S_{i} \cdot S_{j}-\frac{1}{4} n_{i} n_{j}\right),
$$

where $C_{i \sigma}$ and $C_{i \sigma}^{+}$are the Fermi operators, $S_{i}$ are spin operators, $n_{i \sigma}=C_{i \sigma}^{+} C_{i \sigma}$ is a number of electrons at site $i$ with spin $\sigma$, and $n_{i}$ is a total number of electrons at the site.

The very complicated expression for the Hamiltonian, especially for the kinetic term, demands the use of convenient variables. Such variables are just the $X$-operators [2], and in their terms the Hamiltonian is written rather simply. The perturbation theory in the form of the diagram technique for the Hubbard operators was constructed first for the general Hubbard model [3]. In the series of papers [4-9] we have shown that such a technique is especially effective for the $t-J$ model since the number of possible on-site states reduces from 4 to 3 because the pairs of electrons are excluded. The main goal of this paper is to present systematically results of the statistical mechanics $t-J$ model study on the basis of the diagram technique for the Hubbard operators and on the basis of various approximations suggested by us for the description of a wide set of the physical properties of the model such as magnetism, electron states, superconductivity.

In the terms of $X$-operators the Hamiltonian (1.1) has a quadratic form. It is convenient to introduce also a linear term with chemical potential $\mu$ and external magnetic field $h$. Then

$$
\mathcal{H}=\mathcal{H}_{0}+\mathcal{H}_{\text {int }}
$$

where

$$
\begin{aligned}
& \mathcal{H}_{0}=\sum_{i \sigma} \varepsilon_{\sigma} X_{i}^{\sigma \sigma}, \\
& \mathcal{H}_{\text {int }}=t \sum_{i j \sigma} X_{i}^{\sigma 0} X_{j}^{0 \sigma}+J \sum_{i j}\left(X_{i}^{-+} X_{j}^{+-}-X_{i}^{++} X_{j}^{--}\right) .
\end{aligned}
$$

Here $\varepsilon_{\sigma}=-\mu-\sigma \frac{h}{2}$ are the energies of single site states for two spin projections $\sigma$.

Let us introduce a set of the Green functions, determined on Fermi-like $\left(X_{i}^{0 \sigma}, X_{i}^{\sigma 0}\right)$ and Bose-like $\left(X_{i}^{+-}, X_{j}^{-+}\right)$operators and also on the linear combinations $M_{i}=X_{i}^{++}-X_{i}^{--}, N_{i}=X_{i}^{++}+X_{i}^{--}$of the diagonal operators $X_{i}^{\sigma \sigma}$ :

$$
\begin{aligned}
& \mathcal{G}_{\sigma}\left(i \tau, i^{\prime} \tau^{\prime}\right)=-\left\langle T X_{i}^{0 \sigma}(\tau) X_{i^{\prime}}^{\sigma 0}\left(\tau^{\prime}\right)\right\rangle, \\
& \mathcal{D}_{\perp}\left(i \tau, i^{\prime} \tau^{\prime}\right)=-\left\langle T X_{i}^{+-}(\tau) X_{i^{\prime}}^{-+}\left(\tau^{\prime}\right)\right\rangle, \\
& \mathcal{D}_{\|}\left(i \tau, i^{\prime} \tau^{\prime}\right)=-\left\langle T M_{i}(\tau) M_{i^{\prime}}\left(\tau^{\prime}\right)\right\rangle, \\
& \mathcal{D}_{N}\left(i \tau, i^{\prime} \tau^{\prime}\right)=-\left\langle T N_{i}(\tau) N_{i^{\prime}}\left(\tau^{\prime}\right)\right\rangle
\end{aligned}
$$

Here $\mathcal{G}_{\sigma}$ describes an electron propagation, $\mathcal{D}_{\perp}$ and $\mathcal{D}_{\|}$are the Green functions for transverse and longitudinal spin components, and $\mathcal{D}_{N}$ is density-density Green function. All definitions are standard.

Details of the diagram technique for Hamiltonian (1.2)-(1.3) have been described in papers [4-6]. Its elements are fermion $G_{\sigma}^{0}$ and boson $D^{0}$ Green lines 
denoted by solid line (with light and dark arrows for up and down spin projection $\sigma= \pm$ ), dashed lines for $D^{0}$ and cumulants as well, corresponding to the a veraged products of the diagonal operators. Coupling constants are denoted by wavy and dotted lines for kinetical and exchange parts of the Hamiltonian, respectively.

Bare Green functions are on-site quantities, but propagator Green functions $G_{\sigma}^{0}\left(k, \mathrm{i} \omega_{n}\right)$ can be taken in the "Hubbard-I" approximation

$$
G_{\sigma}^{0}\left(k, \mathrm{i} \omega_{n}\right)=\frac{1}{\mathrm{i} \omega_{n}-\xi_{\sigma}(k)+\mu}, \quad \xi_{\sigma}(k)=\left(1-n_{-\sigma}\right) \varepsilon(k),
$$

where $\varepsilon(k)=t \sum_{\Delta} \mathrm{e}^{\mathrm{i} k \cdot \Delta}$ is the bare electron spectrum (without Coulomb interaction).

Hereafter, we shall consider three types of approximations: the generalized random phase approximation (GRPA), the meanfield approximation (MF) and low density (for electrons or holes) approximation (LD). GRPA will be used for calculation of the dynamic magnetic and dielectric susceptibilities in paramagnetic phase, MF - for description of the magnetically ordered phases, and LD - for the study of ground state of the saturated ferromagnetism. The results obtained with the use of the first two approaches should be valid in a wide range of electron concentrations $0<n<1$ excluding perhaps only the edges of this interval, where one can use LD approximation either for electrons $(n \ll 1)$ or for holes $(1-n \ll 1)$.

\section{The generalized random phase approximation}

Similarly to the theory of ordinary Fermi systems where RPA is used being an approximation taking into account only the loop types of diagrams, we introduce GRPA where selection of diagrams is done on the same principle $[4,5]$. The difference is that the loops in the Fermi liquid are constructed from free electron Green lines, while for $t-J$ model the loops include the "Hubbard-l" approximation for Green lines. The other difference comes from more complicated commutator relations for the $X$-operators leading to four different electronic loops [4]:

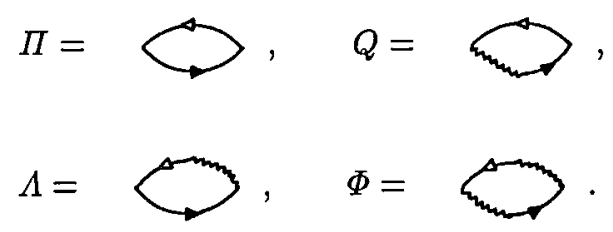

Each of them is a function of 4-momentum $k=\left\{k, i \omega_{n}\right\}$. After summing over discrete frequencies with the use of expression (1.5) for an electron line the loops are expressed through the standard type of sum over space momentum $k$ :

$$
\left|\begin{array}{c}
\Pi(k) \\
Q(k) \\
\Lambda(k) \\
\Phi(k)
\end{array}\right|=\frac{1}{N} \sum_{\boldsymbol{q}}\left|\begin{array}{c}
1 \\
\varepsilon(\boldsymbol{q}) \\
\varepsilon(\boldsymbol{q}-k) \\
\varepsilon(\boldsymbol{q}) \varepsilon(\boldsymbol{q}-k)
\end{array}\right| \frac{f\left[\xi_{\uparrow}(q-k)\right]-f\left[\xi_{\downarrow}(q)\right]}{\mathrm{i} \omega_{n}+\xi_{\uparrow}(\boldsymbol{q}-k)-\xi_{\downarrow}(\boldsymbol{q})} .
$$


Diagrams for the spin Green function $\mathcal{D}_{\perp}$ are chains consisting of loops (2.1) in different combinations. Summing them up we find the dynamic magnetic susceptibility [4]:

$$
\chi(k)=\frac{\chi_{0}(k)}{(1-\Lambda(k))(1-Q(k))+\chi_{0}(k)(\Phi(k)+J(k))} .
$$

Here $\chi_{0}(k)$ has a meaning of "bare" susceptibility

$$
\chi_{0}(k)=\frac{n_{0}}{2 T} \delta_{\omega_{k}, 0}-\Pi(k) \text {. }
$$

It contains two contributions: quasistatic part of the Curie-type $\propto 1 / T$ and the Pauli-type weakly depending on temperature. The factor $n_{0}$ is a function of parameter $\mu / T$ :

$$
n_{0}=\frac{2 \mathrm{e}^{\mu / T}}{1+2 \mathrm{e}^{\mu / T}} .
$$

It changes considerably in the vicinity of $\mu=0$ (energy origin is in the centre of the bare band) from $n_{0}=0$ when $\mu<0$ to $n_{0}=1$ when $\mu>0$. Thus, expressions (2.4) and (2.5) show that when electron concentration varies from 0 to 1 , the system undergoes the crossover from the pure itinerant magnetism to the magnetism with localized magnetic moments. It occurs when critical concentration $n_{\mathrm{c}}$ is such that $\mu\left(n_{\mathrm{c}}\right)=0$. For the symmetrical spectrum $n_{\mathrm{c}}=2 / 3[3,4]$.

From the expression (2.3) there follows an equation for the boundary of the paramagnetic phase stability due to appearance of magnetic ordering with the wave vector $k$ :

$$
(1-\Lambda(k, 0))(1-Q(k, 0))+\left(n_{0} / 2 T-\Pi(k, 0)\right)(\Phi(k, 0)+J(k))=0 .
$$

Here all quantities (1.2) are taken at zero frequency. Magnetic phase diagram following from equation (2.6) will be discussed in Sec. 3 .

The dielectric susceptibility $\chi_{N}(k)$ being actually the Green function $\mathcal{D}_{N}(k)$, is given by formulas similar to (2.3) and (2.4):

$$
\begin{aligned}
& \chi_{N}(k)=\frac{\chi_{0 N}(k)}{(1+\Lambda(k))(1+Q(k))+\chi_{0 N}(k)(\Phi(k)-J(k))}, \\
& \chi_{0 N}(k)=\frac{n_{0}\left(1-n_{0}\right)}{2 T} \delta_{\omega_{k}, 0}-\Pi(k) .
\end{aligned}
$$

We present results of calculations for the Green function $\mathcal{G}_{\sigma}[6,10]$ :

$$
\mathcal{G}_{\sigma}(k)=\frac{1-n_{-\sigma}+\Delta_{\sigma}(k)}{\mathrm{i} \omega_{k}-\xi_{\sigma}(k)-\Sigma_{\sigma}(k)+\mu},
$$

where

$$
\begin{aligned}
& \Delta_{\sigma}(k)=\sum_{q}\left[\frac{A(k q)}{d(q)}+\frac{A_{N}(k q)}{d_{N}(q)}\right] G_{-\sigma}(q+k), \\
& \Sigma_{\sigma}(k)=\sum_{q}\left[\frac{V(k q)}{d(q)}+\frac{V_{N}(k q)}{d_{N}(q)}\right] G_{-\sigma}(q+k)
\end{aligned}
$$

present the terminal and self-energy parts of the Green function $\mathcal{G}_{\sigma}(k)$. In these expressions $d(q)$ and $d_{N}(q)$ denote denominators of expressions (2.3) and (2.7) for 
susceptibilities $\chi(q)$ and $\chi_{N}(q)$ respectively. The amplitudes in formulas for $\Delta_{\sigma}(k)$ and $\Sigma_{\sigma}(k)$ are expressed through the quantities $\Pi, \Lambda, Q$ and $\Phi$ :

$$
\begin{aligned}
& A(k, q)=\frac{3}{2}\left\{\chi_{0}(q)[\varepsilon(k+q)+\Phi(q)+J(q)]-\Lambda(q)[1-Q(q)]\right\}, \\
& V(k, q)=\frac{3}{2}\left\{\chi_{0}(q) \varepsilon(k) \varepsilon(k+q)-\varepsilon(k)[1-Q(q)]\right. \\
&-\varepsilon(k+q)[1-\Lambda(q)]-\Phi(q)-J(q)\} .
\end{aligned}
$$

Similar expressions exist for amplitudes $A_{N}(k q)$ and $V_{N}(k q)[6,10]$.

Therefore, the electron and spin Green functions in GRPA are expressed through four quantities $\Pi(k), \Lambda(k), Q(k)$ and $\Phi(k)$, determined by equations (2.1) with fermion Green function $G_{\sigma}(k)$ yielding Dyson equation:

$$
G_{\sigma}(k)=\frac{1}{i \omega_{k}-\xi_{\sigma}(k)-\Sigma_{\sigma}(k)+\mu} .
$$

In the paramagnetic phase a spin index should be omitted. The magnitude $G(k)$ and four loops $\Pi, \Lambda, Q$ and $\Phi$ depending on it should be determined self-consistently. Let us consider the system near magnetic phase transition. Being in paramagnetic phase we assume the conditions

$$
\frac{T-T_{\mathrm{m}}}{T_{\mathrm{m}}} \ll 1, \quad \frac{T_{\mathrm{m}}}{\mu} \ll 1,
$$

then quasistatic contribution from magnetic fluctuations dominates in expressions (2.11), and Eq. (2.11) reduces to the following:

$$
\Sigma\left(k, \mathrm{i} \omega_{k}\right)=\frac{T}{N} \sum_{\boldsymbol{q}} \frac{3}{2} \varepsilon(k) \varepsilon(k+q) \chi(\boldsymbol{q}, 0) G\left(k+q, \mathrm{i} \omega_{k}\right) .
$$

Near ferromagnetic instability the magnetic susceptibility $\chi(q, 0)$ has a sharp maximum in the vicinity of $q=0$, therefore the smoothly varying function can be taken out from the sum in (2.14). Then we come to a quadratic equation for $\Sigma$ [10]:

$$
\Sigma(k, \omega)=\frac{b \varepsilon^{2}(k)}{\omega-\xi(k)-\Sigma(k, \omega)+\mu} .
$$

In our way from (2.14) to (2.15) we did an analytical continuation onto a real axis $\mathrm{i} \omega_{k} \rightarrow \omega+\mathrm{i} \delta$ and introduced a notation

$$
b=\frac{3}{2} T \frac{1}{N} \sum_{\boldsymbol{q}} \chi(\boldsymbol{q}, 0) \text {. }
$$

Frequency dependence of $G$ is presented in Fig. 1. Here

$$
\omega_{1,2}(k)=\xi(k)=2 \sqrt{b} \varepsilon(k) .
$$

We see that the Green function has a nonquasiparticle (incoherent) character because of ferromagnetic fluctuations. The magnitude $b$ is the measure of fluctuations, it is the measure of incoherency as well. When $b \rightarrow 0$ we have $\omega_{1} \rightarrow \omega_{2}$, therefore the imaginary part of $G$ reduces to $\delta$-peak and we return to the "Hubbard-I" approximation. The parameter $b$ is the guiding parameter, transferring the system from the Fermi liquid regime to the regime with strong correlations where the electronic states have incoherent character. 


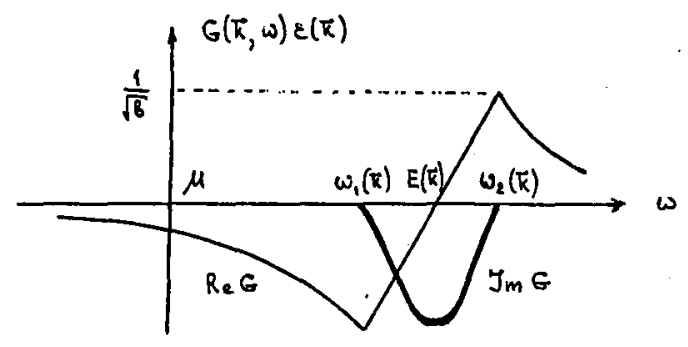

Fig. 1. Real (thin line) and imaginary (thick line) parts of the Green function $G(k, \omega)$ near a ferromagnetic phase transition.

Let us calculate $b$. Near the ferromagnetic phase transition only small vicinity of $q=0$ is essential, that is why

$$
\chi(q, 0) \approx \frac{n_{0}}{\alpha+\beta q^{2}},
$$

with $\alpha \propto T-T_{k}$ and $\beta>0$. Integrating in (2.16) over $q$, we obtain

$$
b=n_{0} \frac{3 T K a^{3}}{4 \pi \beta}\left(1-\frac{1}{K \xi} \arctan K \xi\right),
$$

where $\xi=\sqrt{\beta / \alpha}$ is a correlation length, and $K$ is a cut-off parameter for integration over $q$. Since in the order of magnitude $\beta \sim T_{k} a^{2}$, we have $b \leq 1$. Notice that $b$ is proportional to the factor $n_{0}(2.5)$. Due to its step-like behaviour with chemical potential changing, parameter $b$ does not vanish in the region $n>n_{\mathrm{c}}$ corresponding to the regime with localized magnetic moments. Thus in the vicinity of $n_{\mathrm{c}}$ the system undergoes the crossover with changing of the magnetic and electronic properties simultaneously, transferring from Fermi-liquid behaviour to the regime with strong electron correlations. Processes of inelastic scattering on dynamic fluctuations occur monotonously in the vicinity of $n_{c}$, that is why they can be neglected in the first approximation.

From definition (2.16) it follows that $b$ is actually mean-square static fluctuation of spin at a lattice site. In the self-consistent fluctuation theory [12] in a system with weak Coulomb interaction the similar parameter responsible for the Curie type contribution to $\chi(q, 0)$ appears, that is responsible for the appearance of localized magnetic moments. Similar situation takes place near the antiferromagnetic phase transition [10].

\section{The mean field approximation}

For description of magnetically ordered phase usually the mean field approximation is used, which allows to obtain the equations for the order parameters [11]. Since the $t-J$ model displays simultaneously the features of itinerant and localized magnetism, it is likely to expect the appearance of two coupled order parameters: $m$ - the magnetization of a sublattice and $\Delta$ - the gap in the electron spectrum due to interaction of carriers with the magnetic order. 
Let us determine fermion Green function $G_{\sigma}(k)$ with the help of the following approximation for the self-energy:

$$
\sum_{1}=\operatorname{arma}-\eta_{\alpha}
$$

(similar equation for $\sum_{\downarrow}$ ). The first graph corresponds to the "Hubbard-I" approximation, the next two graphs do not depend on 4-momentum, in the spirit of the mean field approximation. The Green line in the second graph should be considered as an exact one. Thus (3.1) presents a self-consistency equation for $G_{\sigma}(k)$.

Cumulants in (3.1) should be considered as dressed with the help of analogous graphs. A diagram set, for example, for $\left\langle X_{i}^{++}\right\rangle$should have the following structure:

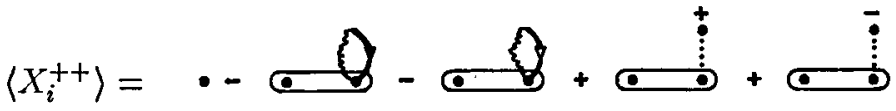

$$
\begin{aligned}
& \ldots+Q-Q
\end{aligned}
$$

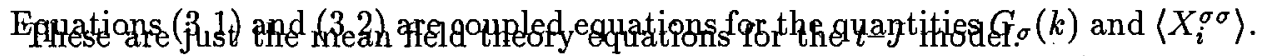

Let us study first a ferromagnetic phase. With the help of Eqs. (3.1) and (3.2) we can write a system of equations for three quantities:

$$
\Delta=\Delta_{\downarrow}-\Delta_{\uparrow}, \quad m=\left\langle X_{i}^{++}\right\rangle-\left\langle X_{i}^{--}\right\rangle, \quad n=\left\langle X_{i}^{++}\right\rangle+\left\langle X_{i}^{--}\right\rangle,
$$

where $\Delta_{\sigma}$ denotes the self-energy part (3.1) which does not depend on $k$ [11]:

$$
\begin{aligned}
\Delta= & \frac{1}{N} \sum_{k} \varepsilon(k)\left\{f\left[\xi(k)+\frac{\Delta-m \varepsilon(k)}{2}\right]-f\left[\xi(k)-\frac{\Delta-m \varepsilon(k)}{2}\right]\right\} \\
& -z J m, \\
m= & -\frac{1}{N} \sum_{k}\left\{f\left[\xi(k)+\frac{\Delta-m \varepsilon(k)}{2}\right]-f\left[\xi(k)-\frac{\Delta-m \varepsilon(k)}{2}\right]\right\} \\
& +n_{0}\left(x, \frac{\Delta}{2 T}\right) \tanh \frac{\Delta}{2 T}+\left[f\left(\frac{\Delta}{2}\right)-f\left(-\frac{\Delta}{2}\right)\right], \\
n= & \frac{1}{N} \sum_{k}\left\{f\left[\xi(k)+\frac{\Delta-m \varepsilon(k)}{2}\right]+f\left[\xi(k)-\frac{\Delta-m \varepsilon(k)}{2}\right]\right\} \\
& +n_{0}\left(x, \frac{\Delta}{2 T}\right)-\left[f\left(\frac{\Delta}{2}\right)+f\left(-\frac{\Delta}{2}\right)\right],
\end{aligned}
$$

where we introduced

$$
n_{0}(x, \eta)=\frac{2 \mathrm{e}^{x} \cosh \eta}{1+2 \mathrm{e}^{x} \cosh \eta}
$$

which transfers to the quantity $n_{0}(2.5)$ when $\Delta=0$. Equations (3.3) and (3.4) present equations for the order parameters $\Delta$ and $m$, while (3.5) - for chemical potential $\mu$.

Let us linearize equations (3.3) and (3.4) with respect to $\Delta$ and $m$. Then they can be written in the form

$$
[1-\Lambda(0,0)] m=\left[\frac{n_{0}}{2 T}-\Pi(0,0)\right] \Delta,
$$




$$
[1-\Lambda(0,0)] \Delta=-[\Phi(0,0)+z J] m,
$$

where we introduced the definitions $\left(f^{\prime}(\xi)\right.$ means a derivative of $f(\xi)$ with respect to $\xi)$ :

$$
\{\Pi(0,0), \Lambda(0,0), \Phi(0,0)\}=\frac{1}{N} \sum_{k}\left\{1, \varepsilon(k), \varepsilon^{2}(k)\right\} f^{\prime}(\xi(k)) .
$$

These quantities are particular values of quantities (1.2) at zero momentum and zero frequency. From (3.7) the equation follows:

$$
[1-\Lambda(0,0)]^{2}+\left[\frac{n_{0}}{2 T}-\Pi(0,0)\right][\Phi(0,0)+z J]=0 .
$$

It determines the phase transition line into paramagnetic phase. It coincides with general equation (2.6) determining the line of paramagnetic phase stability relative to the ferromagnetic ordering. Coincidence of these equations is a sign of the second order transition. From (3.9) an expression for the Curie temperature follows [11]:

$$
T_{k}=\frac{n_{0}}{2} \frac{-\Phi(0,0)-z J}{[1-\Lambda(0,0)]^{2}-\Pi(0,0)[\Phi(0,0)+z J]} .
$$

From expression (3.8) one can see that the denominator in (3.10) is positive and $\Phi(0,0)<0$. Thus formula $(3.10)$ shows that ferromagnetic ordering has the kinetical nature and the exchange interaction hinders it.

Let us study now an antiferromagnetic phase with the wave vector $Q=$ $(\pi \pi \pi) \frac{1}{a}$. The local magnetization alternates from one site to another according to the factor $P_{i}=\mathrm{e}^{-\mathrm{i} \boldsymbol{Q} \cdot \boldsymbol{R}_{i}}$; for this reason an order parameter $m$ should be introduced by the relation:

$$
\left\langle X_{i}^{++}\right\rangle-\left\langle X_{i}^{--}\right\rangle=m p_{i}
$$

Due to that Green function $G_{\sigma}(k)$ determined by the mean field equations (3.1)-(3.2) is the $2 \times 2$ matrix [11]:

where

$$
\hat{G}_{\sigma}(k)=\frac{1}{d_{A}(k)}\left(\begin{array}{cc}
\mathrm{i} \omega_{k}+\mu+(1-n / 2) \varepsilon(k) & \sigma\left[-\Delta-\frac{1}{2} m \varepsilon(k)\right] \\
\sigma\left[-\Delta+\frac{1}{2} m \varepsilon(k)\right] & \mathrm{i} \omega_{k}+\mu-(1-n / 2) \varepsilon(k)
\end{array}\right),
$$

$$
\begin{aligned}
& d_{A}(k)=\left(i \omega_{k}+\mu\right)^{2}-E^{2}(k), \\
& E(k)=\sqrt{\tilde{\xi}^{2}(k)+\Delta^{2}}, \quad \tilde{\xi}(k)=\sqrt{(1-n / 2)^{2}-\frac{1}{4} m^{2}} \varepsilon(k) .
\end{aligned}
$$

The order parameters $\Delta$ and $m$ yield the equations

$$
\begin{aligned}
\Delta= & \frac{1}{2} m z J+\frac{1}{4} m \frac{1}{N} \sum_{k} \varepsilon^{2}(k) \frac{f[E(k)]-f[-E(k)]}{E(k)}, \\
m= & n_{0}\left(x, \frac{\Delta}{T}\right) \tanh \frac{\Delta}{T}+f(\Delta)-f(-\Delta) \\
& -\Delta \frac{1}{N} \sum_{k} \frac{f[E(k)]-f[-E(k)]}{E(k)} .
\end{aligned}
$$


Linearization of these equations leads to the following equation for boundary of an antiferromagnetic phase:

$$
1+\left[\frac{n_{0}}{2 T}-\Pi(Q, 0)\right][\Phi(Q, 0)-z J]=0,
$$

which coincides with the general equation (2.6) when $k=Q$. From here we find the Néel temperature [11]:

$$
\begin{aligned}
& T_{N}=\frac{n_{0}}{2} \frac{z J-\Phi(Q, 0)}{1-\Pi(Q, 0)[\Phi(Q, 0)-z J]} . \\
& \Pi(\boldsymbol{Q}, 0)=\int \mathrm{d} \varepsilon \rho_{0}(\varepsilon) \frac{1}{\xi(\varepsilon)} f[\xi(\varepsilon)], \\
& \Phi(\boldsymbol{Q}, 0)=\frac{-1}{1-n / 2} \int \mathrm{d} \varepsilon \rho_{0}(\varepsilon)(\varepsilon) \varepsilon f[\xi(\varepsilon)] .
\end{aligned}
$$

Since $\Pi(Q, 0)<0$ and $\Phi(Q, 0)>0$, the denominator in (3.16) is positive. From formula (3.16) one can see that the nature of antiferromagnetic ordering is quite different as compared to ferromagnetic one. Antiferromagnetism is caused by the exchange interaction, and electron motion only suppresses it.

In the limit $T \rightarrow 0$ quantities (3.8) are expressed through the density of states $\rho_{0}(\varepsilon)$ in bare spectrum

$$
\{I(0,0), \Lambda(0,0), \Phi(0,0)\}=-\frac{1}{1-n / 2}\left\{1, \tilde{\mu}, \tilde{\mu}^{2}\right\} \rho_{0}(\tilde{\mu}),
$$

where $\tilde{\mu}=\mu(1-n / 2)^{-1}$. Substitution of them into expression (3.10) gives

$$
T_{k}=\frac{n_{0}}{2} \frac{\tilde{\mu}^{2} \rho_{0}(\tilde{\mu})(1-n / 2)^{-1}-z J}{1+(2 \tilde{\mu}+z J) \rho_{0}(\tilde{\mu})(1-n / 2)^{-1}} .
$$

Formulas (3.18) and (3.16) give electron concentration dependence of $T_{K}$ and $T_{N}$, if chemical potential $\mu$ is found as a function of $n$. In the "Hubbard-I" approximation $\mu$ is determined by the equation $[3,4]$ :

$$
n=2\left(1-\frac{n}{2}\right) \int \mathrm{d} \varepsilon \rho_{0}(\varepsilon) f[\xi(\varepsilon)] \text {. }
$$

For the model of constant density of states

$$
\rho_{0}(\varepsilon)=\frac{1}{W}, \quad-\frac{W}{2}<\varepsilon<\frac{W}{2}
$$

( $W=2 z t$ is the band width) from (3.19) the expression follows [3, 4]:

$$
\mu=\frac{3}{2}(n-2 / 3) z t .
$$

Let us determine from Eq. (3.18) $T_{N}$ dependence on $n$. At half filling $(n=1)$

$$
T_{N}^{0}=\frac{1}{2} \frac{z J}{1+\ln 2}
$$

(factor $\ln 2$ has appeared because in Eq. (3.18) a linear term on temperature was taken into account in the quantity $\Phi(Q, 0)$ ). When concentration of holes is small $(1-n \ll 1)$ :

$$
T_{N}=\frac{1}{4} \frac{2 z J-z t(1-n)}{1+\ln 2} .
$$


Due to the large coefficient at $(1-n), T_{N}$ decreases very fast with derivation from half filling. When electron concentration

$$
n_{A}=1-\kappa \quad(\kappa \ll 1)
$$

$T_{N}$ tends to zero. Near $n_{A}, T_{N}$ changes linearly with $n$ :

$$
T_{N}=\frac{1}{2}\left(n-n_{A}\right) z t .
$$

Thus, near $n=1$ antiferromagnetic ordering exists in the interval $n_{A}<n<1$. Ferromagnetic ordering should exist in the interval $n_{\mathrm{c}}<n<n_{\mathrm{F}}$, where $n_{\mathrm{F}}$ is determined by the equation

$$
W \rho_{0}\left(n_{\mathrm{F}} \frac{W}{2}\right)=\kappa \text {. }
$$

These intervals can be overlapped, however near $n=1$ pure antiferromagnetic state should exist. In the overlapped region $T_{N}$ can be much larger than $T_{K}$ at enough large $\kappa$. It is possible to show also that at low temperatures $T \ll T_{N}$, provided $T \ll T_{N}^{0}$, the correlation length for antiferromagnetic fluctuations

$$
\xi \sim \frac{a}{\sqrt{1-n}}
$$

is determined by hole concentration and weakly depends on $T$ [11].

Notice that $T_{K}$ and $T_{N}$ are proportional to the factor $n_{0}$, which according to definition (2.5), strongly changes from 0 to 1 near $n_{\mathrm{c}}$. It is a result of the simplest approximation ignoring fluctuations. In paper [5] we took into account the total set of the Gaussian fluctuations of spin and charge and showed that they do not change the step-like behaviour of $n_{0}$ as a function of $n$. Even the self-consistency in calculating $n_{0}$ does not change qualitatively such a behaviour. Thus, the step-like solution of an equation for $n_{0}$ is a stable with respect to the Gaussian fluctuations. Obviously, $n_{0}$ behaviour near critical concentration $n_{\mathrm{c}}$ is determined by all strongly interacting fluctuations.

There is a tight analogy, with behaviour of a system near localization edge. The quantity $n_{0}$, being a coefficient at a contribution into the magnetic susceptibility $\sim \delta(\omega)$, is similar to a corresponding quantity in the dielectric susceptibility of an inhomogeneous system undergoing localization. In our case in a point $n_{\mathrm{c}}$ we deal with appearance of localized magnetic moments that is a phenomenon of the spin localization. Thus, at present time the question concerning behaviour of the system near the point $n_{\mathrm{c}}$ still remains open.

\section{Low density approximation}

At the edge of the concentration interval $0<n<1$ it is worthwhile to use the low density of electrons $(n \ll 1)$ or holes $(1-n \ll 1)$ approximation. It is possible to use it when studying the saturated ferromagnetic states. It is the well known Nagaoka [13] result that in the limit $U \rightarrow \infty$ at half filling and a single hole the ground state of the Hubbard model should be the saturated ferromagnetism. The question whether this state is stable at finite concentration of holes remains open. 
It is convenient to introduce a hole Green function

$$
\mathcal{G}_{\sigma}^{h}\left(i \tau, i^{\prime} \tau^{\prime}\right)=-\left\langle T X_{i}^{\sigma 0}(\tau) X_{i^{\prime}}^{0 \sigma}\left(\tau^{\prime}\right)\right\rangle
$$

instead of an electron Green function (1.4). Let us calculate the quantities $\mathcal{G}_{\uparrow}^{h}, \mathcal{G}_{\downarrow}^{h}$ and $\mathcal{D}_{\perp}$ under assumption of a ferromagnetic ground state. For study of its stability it is necessary to find an electron(hole)-magnon vertex part. In accordance with the ideology of the low density approximation it is necessary to sum up parallel ladder diagrams for particles: electron (hole) and magnon ones. Since we use the hole representation, when electron concentration is small it is necessary to sum up antiparallel hole-magnon ladders, while at small hole concentration, one must sum up parallel ladders.

As a result we come to the following expressions for hole and magnon Green functions of a system with $n \ll 1$, assuming the ground state to be a saturated ferromagnet:

$$
\begin{aligned}
& \mathcal{G}_{\downarrow}^{h}(k)=-\frac{R(k)}{d_{\mathrm{F}}(k)}, \\
& \mathcal{G}_{\uparrow}^{h}(k)=\frac{1}{\mathrm{i} \omega_{k}+\varepsilon(k)-\mu}, \\
& \mathcal{D}_{\perp}(k)=\frac{n+\Delta(k)}{\mathrm{i} \omega_{k}-\Pi(k)},
\end{aligned}
$$

In expression (4.2):

$$
d_{\mathrm{F}}(k)=1+S(k)-\varepsilon(k) R(k)
$$

and $R(k)$ and $S(k)$ denote two types of hole-magnon loops

$$
\begin{aligned}
& R(k)=\sum_{q} G_{\uparrow}^{h}(q) D(q-k)= \\
& S(k)=\sum_{q} \varepsilon(q) G_{\uparrow}^{h}(q) D(q-k)=
\end{aligned}
$$

In expression (4.4) the terminal and self-energy parts are equal

$$
\begin{aligned}
& \Delta(k)=\sum_{q} G_{\uparrow}(q+k) R(q) \frac{\varepsilon(q+k)-\varepsilon(q)}{d_{F}(q)}, \\
& \Pi(k)=-\sum_{q} G_{\uparrow}(q+k) \frac{\varepsilon(q+k)-\varepsilon(q)}{d_{F}(q)} .
\end{aligned}
$$

Thus in the saturated ferromagnet state $\uparrow$-electron behaves as a free particle, but $\downarrow$-electron has a nonquasiparticle behaviour. When calculating (4.6) and (4.7) a magnon propagator will be approximated as

$$
D(k)=\frac{1}{\mathrm{i} \omega_{k}-\omega(k)},
$$

where $\omega(\boldsymbol{k})$ is to be defined by a self-consistent equation

$$
\omega(k)=\Pi(k, \omega(k)) \text {. }
$$


Numerical solution of this equation leads to the critical concentration $n_{\mathrm{s}}$ when $\omega(k)$ becomes negative at lower concentrations, therefore the saturated ferromagnetism is unstable. For sc lattice $n_{\mathrm{s}}=0.87$ [7]. This value is not small as it is well assumed from the beginning, but rather corresponds to a small concentration of holes. In this case we should sum up parallel hole-magnon ladders and calculate $\mathcal{G}_{\uparrow}^{h}, \mathcal{G}_{\uparrow}^{h}$ and $\mathcal{D}_{\perp}$. It turned out that these functions match the functions calculated in the limit $n \ll$ 1. It means that we may extrapolate the results (4.2)-(4.5) onto the region $n \rightarrow 1$. Thus, the value $n_{\mathrm{s}}=0.87$ can be considered as an interpolation from low electron concentration limit. The similar calculations for finite $U$ show [14] that the region of saturated ferromagnetism is restricted on the $(\kappa, n)$-plane by a curve, joining points 0.87 and 1 with a maximum in some intermediate point, corresponding to small value $\kappa \approx 0.01$. When $\kappa>0.01$ and $n<n_{\mathrm{s}}$, the nonsaturated ferromagnetism exists. Its boundary was calculated earlier within the framework of GRPA.

\section{Conclusions}

The diagram technique for $X$-operators is a powerful tool for study of strongly correlated systems. We managed to show that when electron concentration approaches the critical value $n_{c}$, the system undergoes the crossover with change of magnetic behaviour from entirely itinerant magnetism to magnetism with localized magnetic moments. Simultaneously, the change of electron states takes place from the quasiparticle type (when $n<n_{c}$ ) to the nonquasiparticle type (when $n>n_{c}$ ). A measure of that change is determined by the mean-square fluctuation of magnetic moment at a lattice site.

We have shown also that under definite conditions a ferromagnetic or antiferromagnetic ordering occur. Ferromagnetism is caused by the motion of carriers in the system, while exchange interaction suppresses it. For antiferromagnetism the situation is quite opposite: it is caused by exchange interaction, and the motion of carriers suppresses this state. Nesting in the electron spectrum does not influence much the Néel temperature near half filling. It turned out that $T_{N}$ decreases fast with deviation from half filling, and the correlation length in nonmagnetic region is determined by hole concentration and weakly depends on temperature. These features of antiferromagnetism in the $t-J$ model are qualitatively similar to those observed in the copper-oxides high- $T_{\mathrm{c}}$ compounds.

Besides just listed results, we obtained the spectrum of spin excitations (spin waves) for both ferromagnetic and antiferromagnetic states by calculating poles of the dynamic transverse susceptibility [11]. The study of superconductivity due to indirect electron interactions via spin and charge fluctuations was done as well, but only for a paramagnetic phase of the system [8,9]. List of problems to be solved for the $t-J$ model in nearest time includes: coexistence of superconductivity and antiferromagnetism, possibilities of existence of modulated phases on the magnetic phase diagram, metal-insulator phase transition.

\section{References}

[1] J. Hubbard, Proc. R. Soc. Lond. A 276, 238 (1963). 
[2] J. Hubbard, Proc. R. Soc. Lond. A 285, 542 (1965).

[3] R.O. Zaitsev, Zh. Eksp. Teor. Fiz. 70, 1100 (1976)(JETP 43, 574 (1976)).

[4] Yu.A. Izyumov, B.M. Letfulov, J. Phys., Condens. Matter 2, 8905 (1990).

[5] Yu.A. Izyumov, B.M. Letfulov, E.V. Shipitsyn, J. Phys., Condens. Matter 4, 9955 (1992).

[6] Yu. A. Izyumov, B.M. Letfulov, E.V. Shipitsyn, M. Bartkowiak, K.A. Chao, Phys. Rev. B 46, 15697 (1992).

[7] Yu.A. Izyumov, B.M. Letfulov, E.V. Shipitsyn, K.A. Chao, Int. J. Mod. Phys. B 6, 3479 (1992).

[8] Yu.A. Izyumov, B.M. Letfulov, Europhys. Lett. 16, 497 (1991).

[9] Yu.A. Izyumov, B.M. Letfulov, Int. J. Mod. Phys. B 6, 321 (1992).

[10] Yu.A. Izyumov, B.M. Letfulov, E.V. Shipitsyn, Zh. Eksp. Teor. Fiz., to be published.

[11] Yu.A. Izyumov, B.M. Letfulov, Zh. Eksp. Teor. Fiz., to be published.

[12] T. Moriya, Spin Fluctuations in Itinerant Electron Magnetism, Springer, Berlin 1985.

[13] Y. Nagaoka, Phys. Rev. 147, 392 (1966).

[14] Yu. A. Izyumov, B.M. Letfulov, E.V. Shipitsyn, Fiz. Met. Metalloved., to be published. 